\title{
Athermal Elastomeric Lens Mount for Space Optics
}

\author{
Hagyong Kihm*, Ho-Soon Yang, II-Kweon Moon, and Yun-Woo Lee \\ Center for Space Optics, Korea Research Institute of Standards and Science, 1 Doryong, \\ Yuseong, Daejeon 305-340, Korea
}

(Received May 19, 2009 : revised June 15, 2009 : accepted June 17, 2009)

\begin{abstract}
We investigated the optimum adhesive thickness for athermalizing an elastomeric lens mount in our space optics application. Theoretical results were compared with finite element solutions using two different models; discrete circular pads and discrete circular pads with columns filling the insertion holes reflecting the reality. A noticeable difference between their optimal thicknesses was observed, and physical interpretation revealed the uncertainty of prevailing athermal equations. A pilot sample was made to check our results and thermo-optical stress was assessed using an interferometer after isothermal load. This study presented insight into preliminary design guidance in elastomeric lens mounting.
\end{abstract}

Keywords: Lens mounting, Optomechanics, Athermalization, Elastomeric adhesive, Finite element analysis

OCIS codes : (200.4880) Optomechanics; (040.6040) Thermal effects; (000.4430) Numerical approximation and analysis; (350.6090) Space optics

\section{INTRODUCTION}

Optical systems suffering large temperature variations, where performance is critical, require athermalization in terms of optical and optomechancal design. This paper is focused on the latter case, specifically mounting individual lenses. Optical mounts, usually metals, have larger CTE (coefficient of thermal expansion) than that of optics and stress at the boundary induces optical surface deformation and internal index change lowering optical performance. Even worse, permanent failure might put at stake the system's survival. There are many ways of mounting lenses, but using elastomeric adhesive and/or flexure mounts are frequently adopted if a harsh environment is inevitable [1]. Flexure mounting, quite reliable but rather complicated, is sometimes not adequate due to system requirements like natural frequency and space confinement [2]. Elastomeric mounts require considerable analytical efforts, contrary to the simple and easy embodiment. Aerial photography and surveillance equipment, however, use this simple method while enjoying the advantage of hermetic sealing.

Finding optimum bondline thickness has been the issue

*Corresponding author: hkihm@kriss.re.kr especially in military and space applications [3]. Herbert summarized useful athermal bondline equations and presented guidelines for athermal mounting [4]. Equation (1) is the so-called Bayar's equation [5], where $\alpha_{G}, \alpha_{M}, \alpha_{e}$ are the CTEs of the lens, mount, and elastomer respectively. Equation (2) includes the Poisson's ratio $\nu_{e}$ and is called the Muench equation [1]. These equations will be used as theoretical estimates throughout this paper.

$$
\begin{aligned}
& t_{e \text { Bayar }}=\left(\frac{D_{G}}{2}\right) \frac{\left(\alpha_{M}-\alpha_{G}\right)}{\left(\alpha_{e}-\alpha_{M}\right)} \\
& t_{e \text { Muench }}=\left(\frac{D_{G}}{2}\right) \frac{\left(1-v_{e}\right)\left(\alpha_{M}-\alpha_{G}\right)}{\left[\alpha_{e}-\alpha_{M}-v_{e}\left(\alpha_{G}-\alpha_{e}\right)\right]}
\end{aligned}
$$

Hatheway showed that at normal adhesive thickness the tensile stiffness of the adhesive is about two hundred times greater than the Young's modulus and suggested using at least three or five elements (for more accurate work) through its thickness in finite element modeling [6]. The benefits of using discrete bond pads instead of full circumferential bonding was also verified [7]. Michels and co-workers addressed the difficulties in finite element modeling and the dependence of adhesive stiffness on 
dimensional aspect ratio and Poisson's ratio [8]. Miller compared the finite element solutions with theoretical equations in three different lens types [9].

This research was initiated as a preliminary study

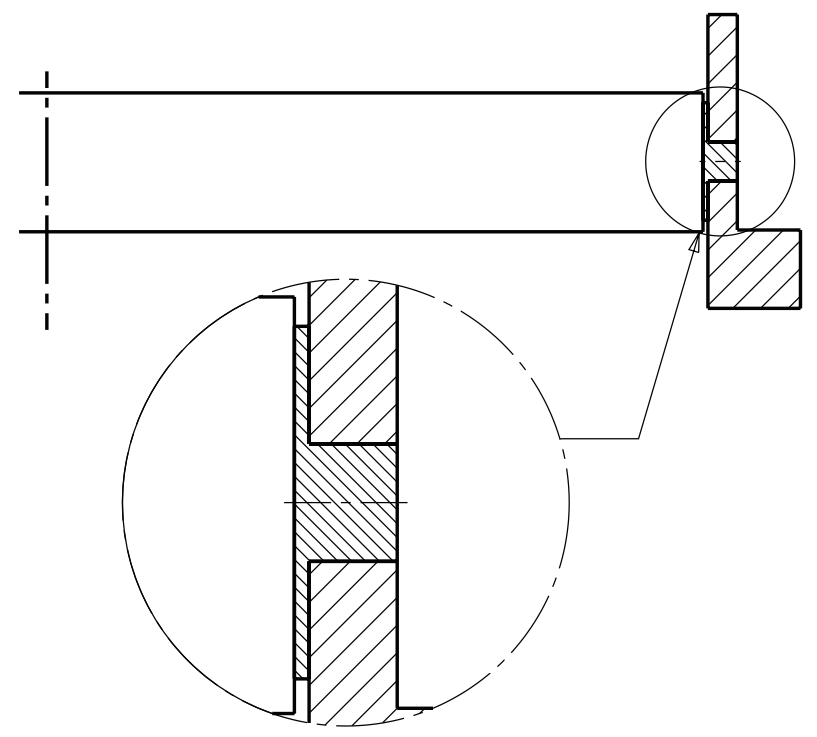

FIG. 1. A pilot sample model made of Schott@ SF6 ( $134 \mathrm{~mm})$ housed in a Ti6Al4V cell $(3 \mathrm{~mm}$ thickness at the rim). The adhesive is $3 \mathrm{M} \otimes$ EC2216 Gray epoxy of which thickness is $0.5 \mathrm{~mm}$ as an initial design step. Adhesive insertion holes $(\varnothing 4 \mathrm{~mm})$ are provided.

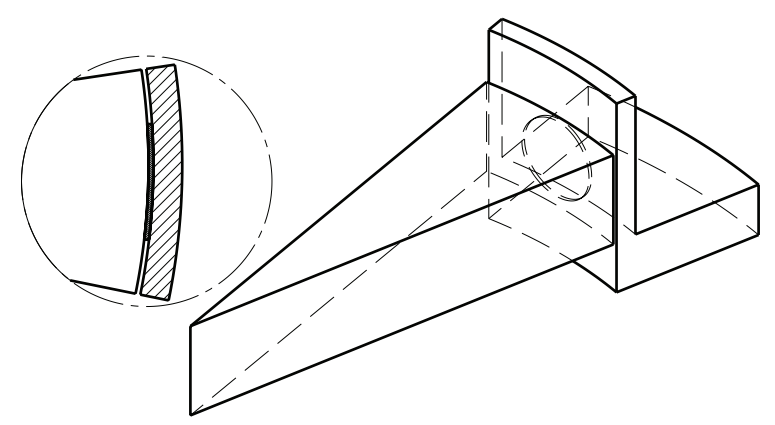

(a)

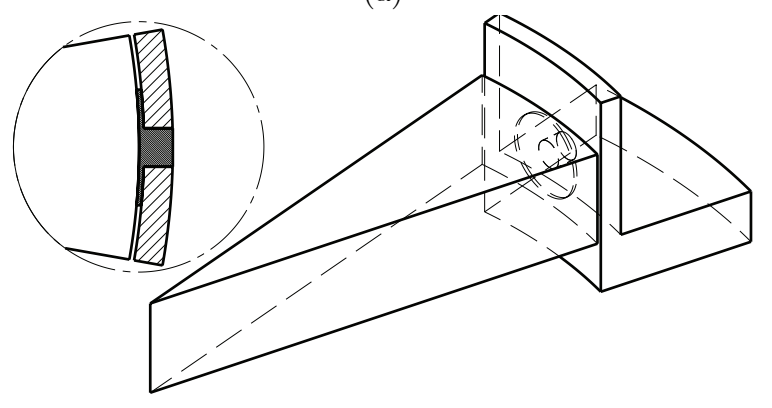

(b)

FIG. 2. Three dimensional model of two different configurations; (a) discrete circular pad ( $\varnothing 12 \mathrm{~mm})$ (ideal case) and (b) discrete circular pad ( $\varnothing 12 \mathrm{~mm}$ ) with a column ( $\varnothing 4 \mathrm{~mm}$, thickness $3 \mathrm{~mm}$ ) filling up to the insertion hole (real case). while tackling athermal lens mounting problems in the infrared optical system for a space satellite mission. A pilot sample was made to understand the physics and verify theoretical and analytical results before applying to the real target at the design stage. Theoretical results based on the pilot sample are compared with finite element solutions using two different models; a simple model of discrete circular pads and a more realistic model of discrete circular pads with columns filling the insertion holes. Theoretical equations only consider stresses in radial directions. But stresses in other directions are quite large at the adhesive boundary. This effect was considered by sensing the Von Mises stress, which is a combination of principal stresses [10]. Plots obtained by varying the bondline thickness of two models gave quite different values regarding optimum solutions. These interesting results are discussed to determine a proper answer to the optimum athermal thickness. Thermooptical stress was assessed using an interferometer after isothermal load. In conclusion, guidance in designing athermal elastomeric lens mounts is presented.

\section{SIMULATION}

\section{Pilot sample configuration}

Difficulties in analyzing adhesive bonds have long been addressed for a number of reasons. The material properties are quite nonlinear depending on the temperature and even show hysteresis. Also the published properties are not always predictable in any cases. Bearing those limitations in mind, we confine our research to a first order approximation. But qualitative information from this study helps in better understanding and finding an optimum design parameter in athermal elastomeric lens mount.

Figure 1 shows the pilot sample model made of a flat mounted within a flanged cell. The flat is AR (anti-reflection) coated on one side and mirror-coated on the other side to facilitate transmissive testing with a visible interferometer. The flat material is Schott SF6, which was selected as a mechanical equivalent for Ge (germanium) due to similar material density and the transparency favoring interferometric testing. Although CTEs of SF6 and Ge are different, SF6 suffices to verify the finite element analysis and derive optimal adhesive thickness applicable to other cases. The cell is flanged just for handling purpose and can be interfaced with environmental testing equipments.

We made two FEA (finite element analysis) models to compare the results with theoretical equations, whereby one can find the adequacy of bondline equations as initial guess tools. Figure 2 depicts two configurations; (a) discrete circular pads and (b) discrete circular pads with columns filling up to insertion hole. Figure 2 (a) is an ideal case where insertion holes are not equipped. Figure 


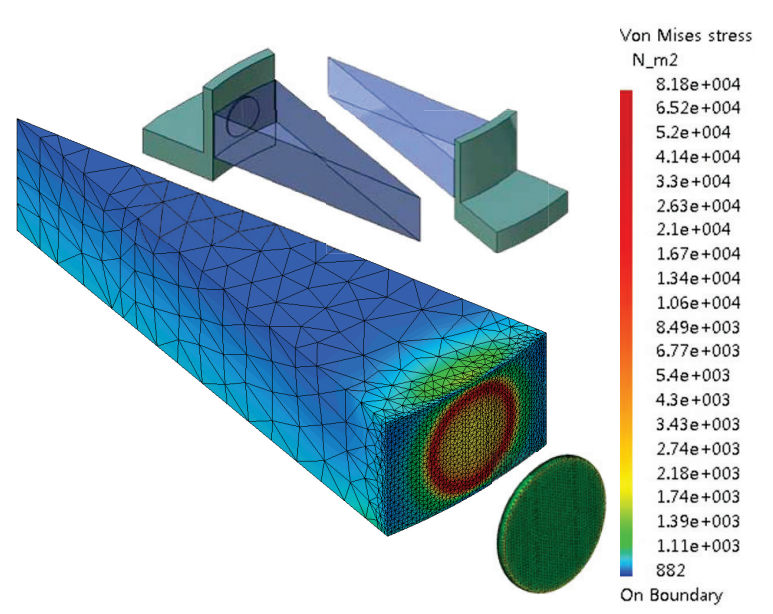

FIG. 3. Stress distribution of ideal circular bonding after isothermal unit temperature load. The adhesive thickness is $0.5 \mathrm{~mm}$.

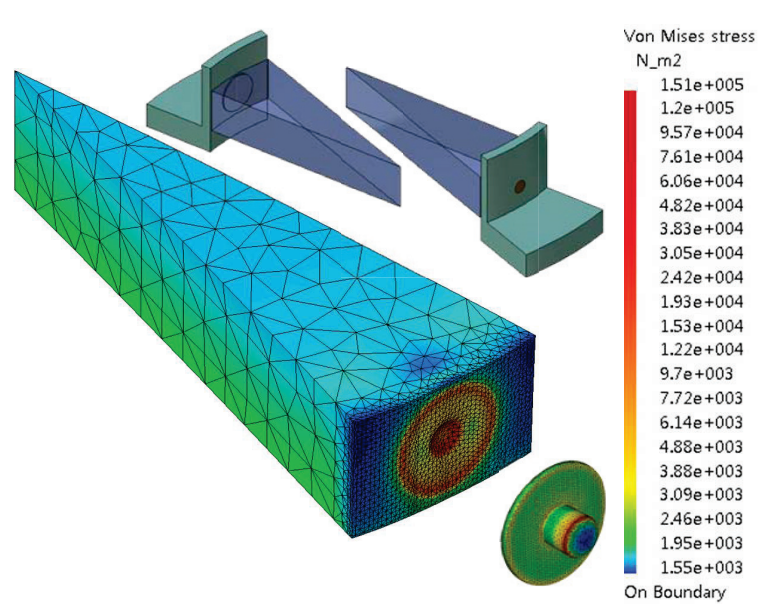

FIG. 4. Stress distribution of circular bonding with an insertion hole after isothermal unit temperature load. The adhesive thickness is $0.5 \mathrm{~mm}$.

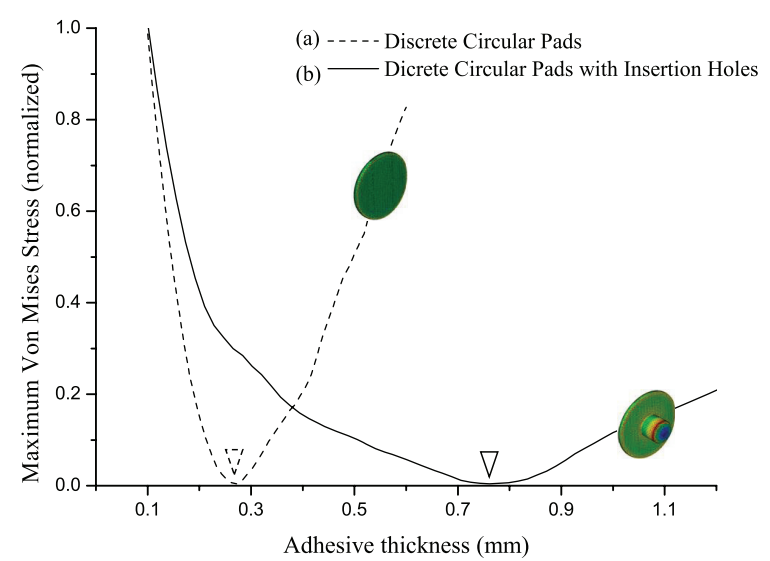

FIG. 5. Maximum Von Mises Stress due to isothermal load with respect to adhesive thickness from (a) discrete circular pads and (b) discrete circular pads with insertion holes as in Figure 2.
2 (b) is a real case having an adhesive insertion hole adaptive to the Luer tip of commercial syringes. They are sliced axisymmetrically with appropriate boundary conditions to reduce computing cost. At least three meshes across the bondlines are guaranteed depending on the thickness.

\section{Simulation results}

Athermal elastomeric lens mounting basically requires CTE differences between lens glass $\left(\alpha_{G}\right)$, cell mount $\left(\alpha_{M}\right)$, and elastomeric adhesive $\left(\alpha_{e}\right)$ to follow the order of $\alpha_{e} \gg \alpha_{M} \gg \alpha_{G}$. In our case, $\alpha_{e}$ of $3 \mathrm{M} 囚 \mathrm{EC} 2216$ is 102 $\operatorname{ppm}\left(10^{-6} /{ }^{\circ} \mathrm{C}\right)$ at room temperature range, $\alpha_{M}$ of Ti6Al4V is $8.8 \mathrm{ppm}$, and $\alpha_{G}$ of Schott@ SF6 is $8.1 \mathrm{ppm}$ [1]. Thus, thermal stresses can be alleviated by adjusting adhesive thickness of which thermal strain fills the gap between lens and mount. Theoretically the radial stress can be zero in one dimensional case, but a point contact is not feasible. Shear stresses play a significant role in actual lens mounting cases, which can only be estimated by FEA. Von Mises stress or effective stress is, therefore, usually adopted as an indicator representing a stress in combination.

Figure 3 shows the FEA result with the configuration of Figure 2 (a), ideal discrete circular pads ( $\varnothing$ $12 \mathrm{~mm}$, t0.5 mm). The ring shaped zone depicts a highly stressed region which coincides with the adhesive circular boundary. The result from Figure 2 (b), discrete circular pads with insertion holes, is shown in Figure 4. The adhesive column filling the insertion hole induces high radial stress contrary to the ideal case in Figure 3. This effect makes the problem more difficult and even unpredictable with simple athermal equations. Given the same adhesive thickness in both cases, Figure 4 shows higher stress concentration. Maximum stress occurs at the inlet of adhesive holes as the adhesive column tries to bulge out with temperature rise. Our concern is, however, the stress at the glass surface which is weak and susceptible to deformation.

We conducted the simulation iteratively on both cases by varying the adhesive thickness and we plotted the maximum Von Mises stress at the glass-adhesive boundary in Figure 5. Figure 5 (a) is from ideal circular pads and (b) is from circular pads with insertion holes. Stress values are normalized respectively with their maximum values for qualitative comparison between two cases, as optimal adhesive thicknesses are of more interest than their absolute stress values. Athermal adhesive thicknesses $\left(t_{e}\right)$ are $0.27 \mathrm{~mm}$ and $0.76 \mathrm{~mm}$ respectively as indicated in Figure 5. Theoretical athermal thickness with equation (1) results in $0.5 \mathrm{~mm}$. Athermal equation (2) considering Poisson's effect, on the other hand, gives $0.2 \mathrm{~mm}$ similar to the result from ideal case (a). But in the real case having adhesive insertion holes, $t_{e}(=0.76$ $\mathrm{mm}$ ) is way beyond the ideal case (a) and theoretical 
Optical Deformation from FEA Result

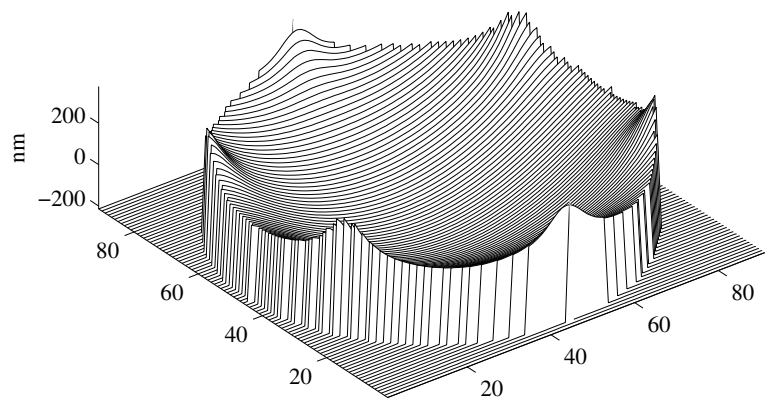

FIG. 6. Optical deformation after $20^{\circ} \mathrm{C}$ isothermal load with non-athermal configuration. Units are arbitrary.

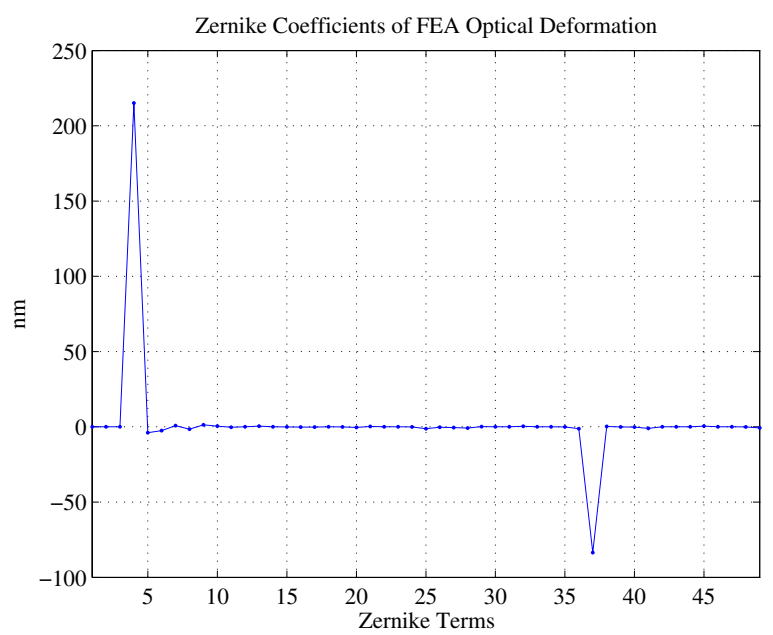

FIG. 7. Zernike coefficients fitted to Figure 6 .

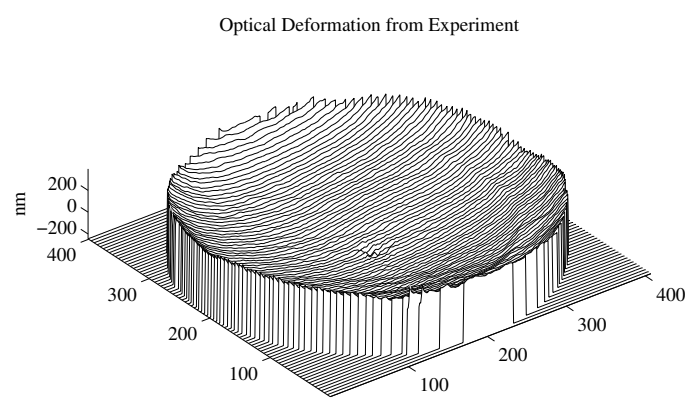

FIG. 8. Interferometric measurement result after $20^{\circ} \mathrm{C}$ isothermal load as in Figure 6. Units are arbitrary.

values from equations (1)-(2). This can be interpreted as the stress from the adhesive column is being dispersed and damped out along the circular pads as it gets thicker. The slope of the curve is high below the athermal value $t_{e}$ and low in the other direction. Yoder [1] mentioned concerning phenomenon that increasing $t_{e}$ beyond the athermal value should tend to decrease temperatureinduced stress because of increased flexibility of the joint.
In this regard, it might be allowable to have large positive tolerance on adhesive thickness.

\section{EXPERIMENT}

\section{Pilot sample configuration}

As an experimental verification, a pilot sample was made as shown in Figure 1. Six insertion holes were provided at every $60^{\circ}$ position. We used commercial Luer-tipped syringes and a syringe pump (TJ-3A, Baoding Longer Precision Pump Co. Ltd.) for minute adhesive volume control after degassing in a vacuum chamber. The bonding area was $12 \mathrm{~mm}$ in diameter and $0.5 \mathrm{~mm}$ in thickness. The viscosity of $3 \mathrm{M} \otimes$ EC2216 was high enough for room temperature curing without a drip. The thickness of the glass was $14 \mathrm{~mm}$ leaving $2 \mathrm{~mm}$ 's margin for the adhesive.

With the stress curve for Schottff SF6 shown in Figure 5 (b), thermo-optical stress would be rarely discernable even with interferometers. In order to exaggerate the effect of non-athermal mounting and compare the FEA results with interferometric measurements, we chose a glass with high $\alpha_{G}$. CDGM® H-ZF7LA having $\alpha_{G}$ of $9.9 \mathrm{ppm}$ was readily available and its optical properties are comparable to Schott® SF6. With this configuration athermal mounting is practically impossible as $\alpha_{G}$ is higher than $\alpha_{M}$. But this setup easily verifies our FEA routines which can be extensively applicable to athermal cases.

\section{Experimental results}

Isothermal load of $20^{\circ} \mathrm{C}$ temperature rise was simulated with the setup of Figure 1, except that the glass material is now CDGM® H-ZF7LA. Even though unit temperature load is preferred for linear analysis, interferometric measurement didn't make substantial results for comparison. Figure 6 shows the optical deformation from FEA results including thickness change and deformation of a mirrored surface. The glass was compressed and six bonding areas are salient. Only $95 \%$ of the aperture was used for analysis to be consistent with interferometric measurement. Figure 7 shows the coefficients of Zernike polynomials [11] fitted to the data in Figure 6. Defocus (4th term) is prominent with $215 \mathrm{~nm}$ and hexafoil (37th term) representing stress concentration at the bonding area is the only high order term with the value of $-83 \mathrm{~nm}$.

The pilot sample was kept in a thermal chamber for 6 hours at $20^{\circ} \mathrm{C}$ above room temperature. Optical deformation was measured with a Fizeau interferometer (Zygo®) GPI 12”) and the result is shown in Figure 8. Manufacturing artifacts are still noticeable even after subtracting the original optical figure before thermal load. And those remnant affected data analysis make it difficult to find the pure thermo-optical stress effects. Zernike 


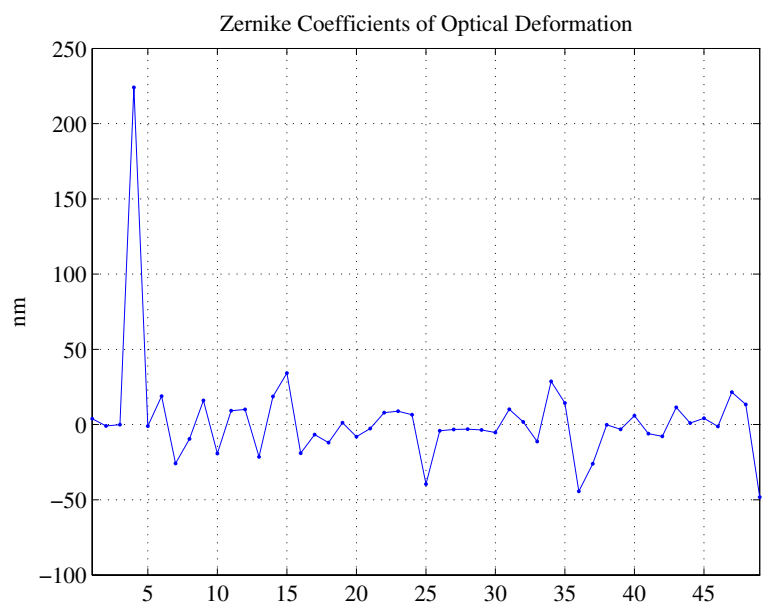

FIG. 9. Zernike coefficients fitted to Figure 8.

coefficients in Figure 9 shows the defocus (4th term) of $224 \mathrm{~nm}$ and the hexafoil (37th term) of $-26 \mathrm{~nm}$, which are the terms of our concern as in Figure 7. The 4th term, the principal contributor, is similar to the FEA result but the 37th term has relatively larger error. The discrepancy could be ascribed to aberrations of the sample optics and better results might be expected with nonaberrant samples. Also the adhesive columns filling the insertion holes had craters at the input port due to surface tension different from the FEA model. Nevertheless, interferometric measurements were in good accordance with FEA results considering the major defocus term.

\section{CONCLUSION}

Athermalization in elastomeric lens mount was investigated to find optimum adhesive thickness in space optics applications. Theoretical equations were compared with FEA results and the validity of those equations was unveiled. The Muench equation showed good estimation, but its value can only be regarded as a lower boundary. Two adhesive configurations, with and without insertion holes, were compared to reflect the difference between real and ideal cases. The difference was noticeable and adhesive insertion holes played significant roles shifting optimum bondline thickness. A pilot sample with nonathermal setup was made to test the FEA routines in an indirect way. Optical deformation obtained by FEA was compared with interferometric measurements and showed relatively good agreement between them except for the artifact. This study suggests using FEA as a preferred way of finding optimum bondline thickness because the solutions largely depend on the actual configurations.

\section{REFERENCES}

1. P. R. Yoder, Mounting Optics in Optical Instruments, 2nd ed. (SPIE Press, Bellingham, WA, USA, 2008), Chapter 3 .

2. J. H. Lee, T. S. Jang, H.-S. Yang, and S.-W. Rhee, "Optical design of a compact imaging spectrometer for STSAT3," J. Opt. Soc. Korea 12, 262-268 (2008).

3. G.-I. Kweon, Y.-H. Choi, and M. Laikin, "Fisheye lens for image processing applications,” J. Opt. Soc. Korea 12, 79-87 (2008).

4. J. J. Herbert, "Techniques for deriving optimal bondlines for athermal bonded mounts," Proc. SPIE 6288, 62880J (2006).

5. M. Bayar, "Lens barrel optomechanical design principles," Opt. Eng. 20, 181-186 (1981).

6. A. E. Hatheway, "Analysis of adhesive bonds in optics," Proc. SPIE 1998, 2-7 (1993).

7. K. B. Doyle, "Athermal design of nearly incompressible bonds,” Proc. SPIE 4771, 296-303 (2002).

8. G. J. Michels, V. L. Genberg, and K. B. Doyle, "Finite element modeling of nearly incompressible bonds," Proc. SPIE 4771, 287-295 (2002).

9. K. A. Miller, "Non-athermal potting of optics," Proc. SPIE 3786, 506-514 (1999).

10. J. E. Shigley and C. R. Mischke, Mechanical Engineering Design, 5th ed. (McGraw-Hill Book Co., New York, USA, 1989), Chapter 6.

11. D. Malacara, Optical Shop Testing, 2nd ed. (John Wiley and Sons, New York, USA, 1992), Chapter 13. 